\title{
The Effectiveness of Cognitive Analytic Therapy on the Severity of Symptoms in Patients with Obsessive-Compulsive Disorder
}

\author{
M. Arab Ameri, A. Yousefi, S. Adl Rad, and F. Rasouli Khorshidi
}

\begin{abstract}
This study was aimed to examine the effectiveness of cognitive analytic therapy on symptoms severity of obsessive-compulsive disorder. This was a empirical study. Twenty patients with obsessive compulsive disorder were selected by inconvenience sampling and randomly assigned to experimental and control group. Participants initially completed the Socio-demographic Questionnaire and interviewed by the Structured Clinical Interview for DSM-IV-TR Axis I Disorders. Then, the Yale-Brown Obsessive-Compulsive Scale was administered in baseline, post-treatment and follow-up. Mixed analysis of variance with repeated measures showed that symptoms severity of obsessive compulsive disorder was significantly decreased at post-treatment and three-month follow-up in the treatment group as compared to the control group $(F 1,18=15.05, P<0.001)$. The interaction of intervention and factor of time resulted in decreased symptoms severity in post-treatment and follow-up $(\mathrm{F} 2,36=22.53, P<0.001)$. The group cognitive analytic therapy is an effective and longstanding therapy for symptoms management of obsessive compulsive disorder.
\end{abstract}

Index Terms-Cognitive analytic, therapy, obsessive-compulsive, disorder.

\section{INTRODUCTION}

Obsessive-Compulsive Disorder (OCD) as a prevalent psychiatric disorder with a life span prevalence of $2 \%$ to $3 \%$, was characterized by recurring intrusive impulses or obsessions and repetitious behavioral or compulsions that habitually takes a chronic course [1]. This disorder has similar clinical rates of prevalence in Iran and causes important trouble or interference with psycho-social functioning [2]. This disorder especially with higher symptoms severity leads to clinically important distress and functional deterioration in general population [3]. Obsessive-compulsive symptom severity was accompanied with diminished psychological health, adverse psycho-social functioning and inappropriate health-related quality of life with an emphasis on the tailored intervention [4]. Psychotherapeutic interventions, in general, are the principal therapeutic procedures for severity and course management of obsessive-compulsive disorder [5].

CAT is a cost-effective therapeutic intervention for

Manuscript received November 2, 2015; revised January 7, 2016.

M. Arab Ameri and F. Rasouli Khorshidi are with Educational Psychology, Islamic Azad University, Babol Brabnch, Iran (e-mail: arabameripsy@yahoo.com, frasouli64@yahoo.com).

A. Yousefi is with Clinical Psychology, Iran (e-mail: azitayusefi@yahoo.com).

S. Adl Rad is with Education and Training Organization, Semnan, Iran (e-mail: adlrad.psy@gmail.com).
Obsessive and compulsive symptoms with additional benefits [6]. CAT was proven to be an effective main intervention causing a rapid rate of improvement recovery from symptoms [7]. In comparison with valid clinical practices, Cognitive Analytic Therapy would operate better meaningfully for diminishing underlying psychopathologies, maladaptive behaviors and to enhance social and vocational functioning [8].

Despite the clearness of the CAT formulation and stages of change for patients with OCD and the acceptance of the CAT procedure, there are too little researches of the effectiveness of this treatment in routine clinical practice [5]. CAT has been recommended as a possible treatment option for OCD as a collection of severe obsessions, severe compulsions, symptom interference, avoidance and symptoms resistance [6]. Up to the present moment, research lines into possible changes in the severity symptoms in patients with obsessive-compulsive disorder after psychotherapeutic intervention has mainly been limited to small and short-term results. This condition has led several researchers to recommend additional investigation on stable and long-term changes of the symptoms on the basis of new directions in psychotherapy [9]. Cognitive analytic therapy attempts to comprehend and improve self limiting and chronic patterns of affective expression, ritual behaviors/tries, and long-standing obsessions, find the principal emotional models of relating to others and one self and their relationship to the patient's presenting difficulty or obvious distress [10]. This study is rare regarding its use of a randomized clinical trial design to investigate the responsiveness of patients with OCD to CAT under routine clinical practice situations, during the therapy course and the follow-up length in Iran.

This study was aimed to examine the effectiveness of cognitive analytic therapy on symptoms severity in patients with obsessive-compulsive disorder in out patient treatment setting.

\section{METHOD}

This study is a clinical trial with a control group that used the quasi-experimental design to examine the differences in the severity of symptoms as dependent variables for patients with obsessive-compulsive disorder before and after one course of group cognitive analytic therapy as independent variable in a systematic manner in terms of three measures of pre-treatment, post-treatment and follow-up.

\section{INTERVENTION}




\section{A. Participants}

Statistical population was patients with obsessive-compulsive disorder who attended to Ravan-Sabz Center of Psychiatric Rehabilitation in Semnan City, Iran from 2014 to 2015. Participants have a range of age from 28 to 40 years old with the mean of $37.65 \pm 3.72$ and were fluent in Persian. All 20 participants met DSM-IV-TR diagnostic criterion for obsessive-compulsive disorder based on the Structured Clinical Interview for DSM-IVTRAx is I Disorders (SCID) and pharmacotherapy was prescribed by psychiatrists in Ravan-Sabz Center. Firstly, among the statistical population, 24patients (12 male and 12 female) with obsessive compulsive disorder were selected by inconvenience sampling and in equivalent sample size randomly assigned to experimental group and control group. In intervention phase, two male and one female were dropped out from treatment group prior to the termination of the study. Also, one male didn't participate in post-test and follow-up in the control group. Therefore, ultimate participants in this study for treatment group was 9 patients (4male and 5 female) and in control group was 11 patients ( 5 male and 6 female) totally including 20participants who stayed throughout the study up to the follow-up.

All participants consumed medications prescribed by psychiatrists for the control of OCD before the study. The Two groups received the same medication so that, its kind, dosage, duration and consumption conditions were controlled by researchers for both groups.

\section{B. Procedure}

Data were collected the routine clinical practice at three points: baseline, discharge of intervention (post-treatment), and three-month follow-up after discharge phase by trained researchers. Participants in the two group initially completed the Socio-demographic Questionnaire and were interviewed by the Structured Clinical Interview for DSM-IVTRAxis I Disorders (SCID) by trained clinicians, then the Yale-Brown Obsessive-Compulsive Scale(Y-BOCS) as a measure of symptoms severity were administered in baseline (pre-treatment), 4 month safterwards (post-treatment) and 3 months after the intervention termination (follow-up). Among two groups, after baseline assessment the treatment group only received 16 sessions of group cognitive analytic therapy (one fifty-minute weekly session) and control group didn't received any intervention, then both groups were assessed by outcome measures in 4 months from baseline and also 3 months later all of them followed by stability of outcome changes.

The intervention performed on the basis of five stages in CAT in 16 sessions as was consistent with the CAT manual [11]. In the final session (sixteen) goodbye letters were exchanged between the patient and therapist that summarized and revealed main changes that had obtained the importance of the termination, and the forward challenges beyond the treatment. At the end of all sessions, the therapist set homework include filling in a scale for depicting frequent maladaptive procedures and drawing patient's procedural life, monitoring the target problems and behaviors/feelings that trigger them by keeping a diary and also tailored techniques for controlling them.

\section{MEASURES}

\section{A. Socio-demographic Questionnaire}

All participants were assessed by a Semi-structured Questionnaire Form that characterizes age, gender, educational level, marital status, occupational status, and previous medical conditions history of the cohorts. In this form, OCD patients were also assessed regarding duration of disorder, kind of obsessions and compulsions, treatment duration, comorbidity and medication taken.

\section{B. The Structured Clinical Interview for DSM-IVTR}

Axis I Disorders (SCID): All participants interviewed by the Structured Clinical Interview for DSM-IV-TR Axis I Disorders as an initial assessment to ensure a diagnosis of OCD. In this study, an experienced psychiatrist supervised the execution of the interview and the attainment to the DSM-IV-TR diagnosis so that prevent diagnostic errors. Internal consistency with Cronbach's $\alpha$ for SCID has been determined in the range of .77 to .88 [12].

\section{Yale-Brown Obsessive Compulsive Severity}

Scale (YBOCS): The Yale-Brown Obsessive-Compulsive Scale (YBOCS) was used to assess obsessive compulsive symptoms severity. The scores of YBOCS severity in a five-point likert-type scale range from 0 (no symptoms) to 4 (severe symptoms), and yield a total severity score(range from $0-40$ ) with a cut point score $\geq 16$ revealing clinically important symptoms [3]. In a sample of 130 adult patients with obsessive- compulsive disorder, the YBOCS-IISeverity Scale demonstrated robust internal consistency $(a=0.89)$, test-retest reliability and inter-rater reliability (intra-class correlations > 0.85). Exploratory factor analysis showed a 2 factor solution in accordance with the Obsessive and Compulsive Severity subscales. Construct and content validity was affirmed by higher correlations with both clinician's assessments of OCD symptom intensity and measures of depressive and worry symptoms [13]. This scale has good test-retest reliability, internal consistency and inter-rater reliability in Iran [14].

\section{Statistical ANALYSIS}

For data analysis, mixed analysis of variance with repeated measures by SPSS-18 software was conducted to compare scores on the OCD severity at baseline (pre-treatment), post-treatment (following the intervention) and follow-up (three-month latter) between two groups.

\section{RESULT}

The mean age of all participants was 37.65 2 3.72(range: 30-45). Also, the mean age for treatment group was $37.78 \pm 5.38$ (range: 31-44) and for control group was $36.36 \pm 5.9$ (range: 30-43). In terms of Tests of Within-Subjects Effects, there are 3 time measurement of OCD severity scores of pretreatment, post-treatment and follow-up (3 months after the treatment). About the Tests of Between- Subjects Effects, treatment group had 9 participants (4 males and 5 females) and control group had 11 participants 
(5 males and 6 females). The means and standard deviations

of OCD severity scores are presented in Table I.

TABLE I: MEAN (SD) OF OCD SEVERITY SCORES FOR PRE-TREATMENT, POST-TREATMENT AND FOLLOW-UP IN GROUPS

\begin{tabular}{|c|c|c|c|c|c|}
\hline variable & group & number & Pre-treatment & Post-treatment & Follow-up \\
\hline & & & Mean(SD) & Mean(SD) & Mean(SD) \\
\hline & treatment & 9 & $29.11(5.58)$ & $17.78(4.15)$ & $14.56(3.91)$ \\
\hline OCD severity & control & 11 & $30.73(5.71)$ & $27.91(5.32)$ & $29.09(7.66)$ \\
\hline & total & 20 & $30.00(5.56)$ & $23.35(6.99)$ & $22.55(9.62)$ \\
\hline
\end{tabular}

TABLE II: RESUltS OF MIXED ANALYSIS OF VARIANCE FOR 3 TIME MEASUREMENT OF OCD SEVERITY SCORES IN TOW GROUPS

\begin{tabular}{|c|c|c|c|c|c|c|}
\hline source & Ss & df & Ms & Partial ETA squared & P & \\
\hline Treatment & 1139.82 & 1 & 1139.80 & 15.05 & 0.001 & \\
\hline error & 1363.47 & 18 & 75.75 & & & \\
\hline factor & 769.90 & 2 & 384.95 & 40.62 & 0.455 \\
\hline Factor*treatment & 427.04 & 2 & 213.52 & 22.53 & 0.005 & 0.693 \\
\hline Error(factor) & 341.20 & 36 & 9.48 & & & \\
\hline
\end{tabular}

Initially, test assumptions were examined. Box's Test of Equality of Covariance Matrices (Box's $M=11.07$ ) showed that the observed covariance matrices of the dependent variables are equal across groups $(\mathrm{F} 6,2068=1.50, p=0.174)$. Also, Levene's Test of Equality of Error Variances showed that the error variance of the dependent variable is equal across groups (for pre-treatment).

$\mathrm{F} 1,18=0.540, \mathrm{p}=0.472$; for post-treatment:

$\mathrm{F} 1,18=1.808, \mathrm{p}=0.195$ and for follow-up:

$\mathrm{F} 1,18=3.734, \mathrm{p}=0.069)$. In addition, results of Mauchly's Test of Sphericity $(0.974, \mathrm{p}=0.797)$ confirmed the Sphericity assumption, that indicate the assumptions for application of mixed analysis of variance are established.

According to mixed analysis of variance by Tests of Between-Subjects Effects (Table II), the reduction in YBOCS total scores was significantly larger overall dependant on the treatment group, so that in the post-treatment and follow-up periods the scores of treatment group was decreased in comparison with control group $(F=15.05$, df1 $=1$, df $2=18$, $P<0.001)$. Also, Bonferroni t test showed that the treatment and the control group don't significantly differ in pre-treatment $(P>0.05)$, while the difference is statistically significant in post-treatment and follow-up. The post-treatment and follow-up means (17.78 and 14.56 respectively) for treatment group were significantly lower than the post- treatment and follow-up means (27.91 and 29.09 respectively) for control group $(p<0.05)$. That is, CAT model was effective in decreased severity of OCD symptoms in the treatment group. In accord with the Tests of Within-Subjects Effects about difference among three-time measurement (factor of time) between the two groups, the YBOCS scores were significantly diminished in the treatment group than in the control group at any time-point following the treatment $(\mathrm{F} 2,36=40.62, P<0.001)$. Also, the mixed analysis of variance revealed that the interaction of treatment (intervention) and treatment period (factor of time) resulted in a decreased YBOCS total scores (F2,36 $=22.53, P<$ 0.001). According to the table 1, compared with baseline (Pretreatment),the YBOCS scores were significantly decreased only in the treatment group in month 3 (post-treatment) and in month 6 follow-up $(P<0.001)$. Results of pair wise comparisons by Bonferroni $t$ test showed that decreasing in the post-treatment and follow-up means (17.78 and 14.56 respectively) in comparison with the pretreatment mean (29.11) was statistically significant in treatment group $(p<0.05)$, but, this mean comparisons $(30.73$, 27.91 and 29.09 respectively for pre-treatment, post-treatment and follow- up) in control group aren't statistically significant $(p>0.05)$ and means of control group in 3 phases didn't significantly change (table 2). Also, these changes from post- treatment to follow-up aren't statistically significant in both groups $(p>0.05)$. In other words, these results are indicating the effectiveness of CAT on reduction of OCD severity. Mean difference of OCD severity in two groups during 3 measurement phases (Pretreatment, Post-treatment and Follow-up) was presented in Fig. 1.

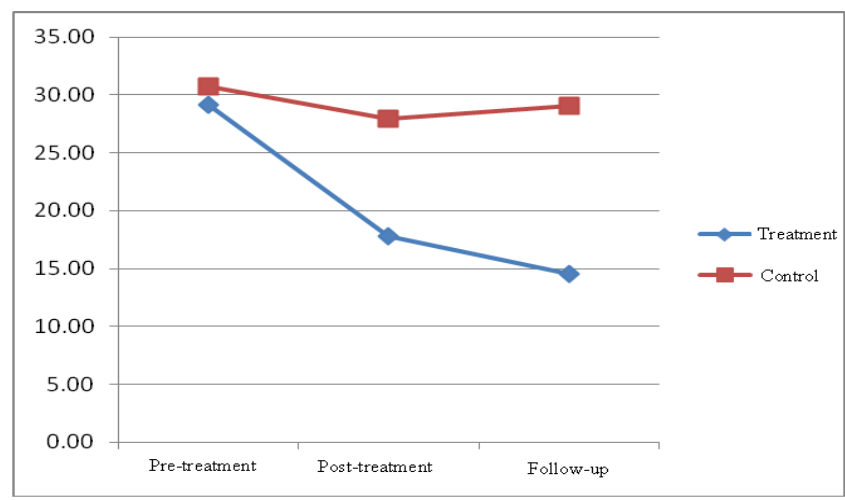

Fig. 1. Mean differences in OCD severity scores in both treatment and control group during three- time measurement.

\section{DISCUSSION}

This study was performed to investigate the effectiveness of cognitive analytic therapy on symptoms severity in patients with obsessive-compulsive disorder in one Iranian outpatient sample. Results showed that between the two groups, the YBOCS scores were significantly lower in the treatment group than in the control group at any time-point after intervention (post-treatment and follow-up). Thus, cognitive analytic therapy is an effective treatment for managing of the symptoms severity in obsessive-compulsive disorder. This study in accordance with previous researches demonstrate that cognitive analytic therapy can be used to control most 
important OCD symptoms with higher therapeutic response and recovery rates, better adherence, decreased OCD symptom severity rapidly, and with improved psycho-social function in patients with OCD [3], [9], [15] showed that CAT with an emphasis on the relational origins, personality characteristics and the background of psychopathology is well capable to reducing the symptoms. Various factors might be accountable for why CAT reduces severity of OCD quickly, although this result is supported with clear evidence. First, CAT may set a suitable therapeutic target order from permanent characteristics of OCD including fear to intrusive thoughts/obsessions and then to compulsions/rituals. Second, the education of disease managing skills may be the proper methods in CAT for treatment of OCD. Fourth, in accord with the model of Dunn, four important ingredients of OCD that can be addressed with CAT recognized as intrusive obsessions, wrong appraisal, being afraid of negative events, and compulsive behaviors [16]. One target of CAT is to break down the reinforcing associations between intrusive obsessions, negative occurrences, and compulsive behaviors, which is obtained by using problem focused and appraisal-focused coping strategies in this treatment model.

In line with other results, our findings indicate that the symptoms severity of OCD can be decreased in the combination of the CAT with pharmacotherapy [15], [17]. According to the opinion of [17], psychotherapies that were based on cognitive-behavior techniques in combination with pharmacotherapy were more effective in symptoms management and quality of life improvement in outpatient and in patient treatment settings for obsessive-compulsive disorder. The present study shows that patients with OCD suffer from high symptoms severity improved over4 months course of CAT and this improvement lasts for 3 months follow-up in comparison with control group. So, the YBOCS scores were significantly lower in the treatment group than in the control group at any time-point after intervention (post-treatment and follow-up). Thus, CAT is an effective treatment for managing of the symptoms severity in OCD. To the best of our understanding, the present study repetitively was administered a psychological intervention in patients with OCD over a 4 month course. Thus, our results enlarge antecedent researches by supplying facts on long-term changes of OCD symptoms. Using a control group, covering the clinical study with a 3-month follow-up, control for medication, using structured interview for OCD diagnosis, using the appropriate measures of OCD severity and selection of the representative sample were the most important strengths in this study. Nonetheless, several important limitations needed to be considered in generalization of the conclusion. First, deductions about causal relationships between the execution of CAT and following out patient treatment, psychosocial improvement, and symptomatic alterations can not be depicted from these findings because of sampling process and inclusion and exclusion criteria. Second, the sample size for group treatment is relatively small, which diminishes the power of the data analysis. Also, data collection was obtained from only one outpatient institution. Thus, a multicenter clinical trial with independent raters and longer-term treatment studies is required to additional determine the efficacy of CAT. Third, lack of detailed information about the rapeuticadherence of participants may result in bias in process of deducting and study report-writing. Finally, lake of clinical significance calculation in this study is an important shortcoming that should be an issue for consideration in future studies.

Future studies are required to further extricate the influence of separate changes in psychopathological course on long-term alterations in the OCD severity. In summary, our preliminary study suggests CAT has a capability for long-standing effective treatment of OCD. Additional multicenter clinical trials and studies with various cultural backgrounds are required.

\section{REFERENCES}

[1] M. Subramaniam, E. Abdin, J. A. Vaingankar, and S. A. Chong, "Obsessive compulsive disorder: Prevalence, correlates, help-seeking and quality of life in a multiracial Asian population," Journal of Social Psychiatry and Psychiatric Epidemiology, vol. 47, no. 12, pp. 2035-2043, Dec. 2012

[2] N. Akrami, M. Kalantari, H. R. Oreyzi, M. R. Abedi, and M. Maroofi, "Comparison of the effectiveness of behavioural-cognitive \& behavioural-metacognitive approaches in patients with obsessive-compulsive disorder (OCD)," Journal of Clinical Psychology, vol. 2, no. 2, pp. 59-71, 2013.

[3] P. Skapinakis, S. Bellos, S. Koupidis, I. Grammatikopoulos, P. N. Theodorakis, and V. Mavreas, "Prevalence and sociodemographic associations of common mental disorders in a nationally representative sample of the general population of Greece," BMC Psychiatry., vol. 13, no. 163, June 2013.

[4] J. S. Abramowitz et al., "Obsessive-compulsive disorder," IEEE Trans. Image Process., vol. 8, no. 374(9688), pp. 491-499, Aug. 2009.

[5] E. Mancuso, A. Faro, G. Joshi, and D. A. Geller, "Treatment of pediatric obsessive-compulsive disorder: a review," Journal of Child and Adolescent Psychopharmacology, vol. 20, no. 4, pp. 299-308, 2010.

[6] C. Protogerou, G. Garyfallos, K. Katsigiannopoulos, V. Bozikas, M. Voikli, and O. Georgiadou, "Evaluation of cognitive-analytic therapy (CAT) outcome in patients with obsessive-compulsive personality disorder," Annals of General Psychiatry., vol. 7, no. 1, pp. 109-111, 2008.

[7] L. Shine and M. Westacott, "Reformulation in cognitive analytic therapy: effects on the working alliance and the client's perspective on change," Psychology and Psychotherapy: Theory, Research and Practice, vol. 83, no. 2, pp. 161-177, 2010.

[8] S. Kellett, D. Bennett, T. Ryle, and A. Thake, "Cognitive analytic therapy for borderline personality disorder: Therapist competence and therapeutic effectiveness in routine practice," Clinical Psychology \& Psychotherapy, vol. 20, no. 3, pp. 216-225, 2013.

[9] J. Hepple, "Cognitive analytic therapy in a group: Reflections on a dialogic approach," British Journal of Psychotherapy, vol. 28, no. 4, pp. 474-495, 2012.

[10] A. Ryle, Cognitive Analytic Therapy: Development in Theory and Practice, Wiley \& Sons, London, 1995.

[11] A. Ryle, Cognitive Analytic Therapy: Active Participation in Change, Wiley \& Sons, London, 1991.

[12] B. M. First, M. Gibbon, and R. L. Spitzer, "User's guide for SCID-I structured clinical interview for DSM-IV-TR axis I disorders, research version," New York: Biometrics Research Department, New York State Psychiatric Institute, 2002.

[13] E. A. Storch, M. J. Larson, L. H. Price, S. A. Rasmussen, T. K. Murphy and W. K. Goodman, "Psychometric analysis of the yale-brown obsessive - Compulsive scale second Edition symptom checklist," Journal of Anxiety Disorders, vol. 24, no. 6, pp. 650-656, 2010.

[14] S. H. Hosseini, M. Zarghami, A. Moudi, and A. R. Mohammadpour, "Frequency and severity of obsessive-compulsive symptoms/disorders, violence and suicidal in schizophrenic patients," Iranian Red Crescent Medical Journal, vol. 14, no. 6, pp. 345-351, 2012.

[15] C. W. Lack, "Obsessive-compulsive disorder: Evidence based treatments and future directions for research," World Journal of Psychiatry., vol. 2, no. 6, pp. 86-90, 2012.

[16] M. A. Dunn, "Cognitive analytic formulation," Clinical Case Formulation: Varieties of Approaches, John Wiley \& Sons, Ltd. 2009. 
[17] E. Hertenstein, N. Thiel, N. Herbst, T. Freyer, C. Nissen, A. K . Külz, and U. V. Herbst, "Quality of life changes following inpatient and outpatient treatment in obsessive-compulsive disorder: a study with 12 months follow-up," Annals of General Psychiatry, vol. 12, no. 4, pp. $1-9,2013$.

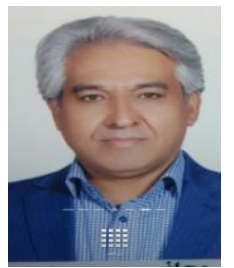

M. Arab Ameri was born on November 24, 1967. He is a $\mathrm{PhD}$ student of educational psychology, Islamic Azad University, BabolBrabnch, Iran from 2015 to 2016.

He worked in education and training organization, Semnan, Iran. He is teaching in Islamic Azad University, Babol Brabnch, Iran and Farhangian University, Semnan, Iran. His research Project: 1) Study prevalence of learning disabilities in primary students (grades second through fifth), Semnan; 2) Study the fields of health, economic and social in indication of disability in Semnan; 3) Compare the evolution of mathematical logic operations in the normal students with learning disabilities in Semnan.

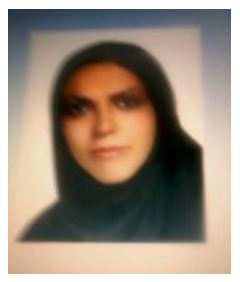

A. Yousefi was born on September 26, 1981. She got the M.A from Clinical Psychology, Semnan, Iran in 2013.

She worked as a manager of Learning Disorders Center Semnan, Iran. She wrote some articles.
S. Adl Rad was born on September 10, 1969. S. Adl Rad got the M.A in psychology, Semnan, Iran in 2015

S. Adl Rad worked as a consultant in the Education and Training Organization, Semnan, Iran. S. Adl Rad's research project: Study prevalence of learning disabilities in primary students (grades second through fifth) in Semnan

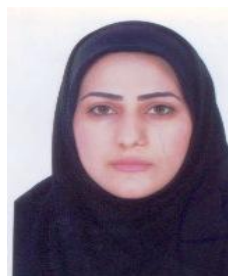

F. Rasouli Khorshidi.was born on April 18, 1985

She is a $\mathrm{PhD}$ student of educational psychology, Islamic Azad University, Babol Brabnch, Iran from 2015 to 2016.

She worked as an employee of governership, Agh Ghalal Golestan, Iran. She is teaching in Islamic Azad University, Babol Brabnch, Iran. She wrote some articles. 